\title{
Slow Electron-Phonon Cooling in Superconducting Diamond Films
}

\author{
Nadezhda Titova, Anna I. Kardakova, Nina Tovpeko, Sergey Ryabchun, Soumen Mandal, Dmitry Morozov, \\ Georgina M. Klemencic, Sean R. Giblin, Oliver A. Williams, Gregory N. Goltsman, and T. M. Klapwijk
}

\begin{abstract}
We have measured the electron-phonon energyrelaxation time, $\tau_{\mathrm{eph}}$, in superconducting boron-doped diamond films grown on silicon substrate by chemical vapor deposition. The observed electron-phonon cooling times vary from $160 \mathrm{~ns}$ at $2.70 \mathrm{~K}$ to $410 \mathrm{~ns}$ at $1.8 \mathrm{~K}$ following a $T^{-2}$-dependence. The data are consistent with the values of $\tau_{\mathrm{eph}}$ previously reported for singlecrystal boron-doped diamond films epitaxially grown on diamond substrate. Such a noticeable slow electron-phonon relaxation in boron-doped diamond, in combination with a high normal-state resistivity, confirms a potential of superconducting diamond for ultrasensitive superconducting bolometers.
\end{abstract}

Index Terms-Superconducting boron-doped diamond, electron-phonon time, superconducting nanobolometers.

\section{INTRODUCTION}

A SUPERCONDUCTING film on a dielectric substrate is a key element of powerful and practically usable radiation detectors such as superconducting single-photon detectors (SSPDs) [1], microwave kinetic inductance detectors (MKIDs) [2] and hot electron bolometers (HEBs) [3]. Each type of detectors has become very prominent in the last decade. SSPDs are the fastest single-photon detectors for photon counting in optical and near infrared range, they are commercially available. MKID is promising detector technology due to the scalability and the simplicity of multiplexing a large number of pixels. HEB as a mixer is the most sensitive low noise devices

Manuscript received September 8, 2016; accepted November 8, 2016. Date of publication December 9, 2016; date of current version February 17, 2017. This work was supported in part by the Ministry of Education and Science, Russia, contracts No. 14.B25.31.0007, in part by the Russian Foundation for Basic Researches under Grant 16-29-11779 ofi-m.

N. Titova, A. I. Kardakova, and N. Tovpeko are with the Physics Department, Moscow State University of Education, Moscow 119435, Russia (e-mail: titovana@mail.ru; kardakova@rplab.ru; mouseninka@mail.ru).

S. Ryabchun and G. N. Goltsman are with the Physics Department, Moscow State University of Education, Moscow 119435, Russia, and also with the Higher School of Economics, National Research University, Moscow 101000, Russia (e-mail: sergey.ryabchun@gmail.com; ggoltsman@hse.ru).

S. Mandal, D. Morozov, G. M. Klemencic, O. A. Williams, and S. R. Giblin are with the School of Physics and Astronomy, Cardiff University, Cardiff CF24 3AA, United Kingdom (e-mail: soumen.mandal@gmail.com; Dmitry. Morozov@astro.cf.ac.uk; georgina.klemencic@gmail.com; giblinsr@cardiff. ac.uk; WilliamsO@Cardiff.ac.uk).

T. M. Klapwijk is with the Kavli Institute of the Nanoscience, Delft University of Technology, 2628 CJ Delft, The Netherlands, and also with the Physics Department, Moscow State University of Education, Moscow 119435, Russia (e-mail: t.m.klapwijk@ tudelft.nl).

Color versions of one or more of the figures in this paper are available online at http://ieeexplore.ieee.org.

Digital Object Identifier 10.1109/TASC.2016.2638199 for THz-range radiospectroscopy and as a direct power detector (nano-HEBs) makes a promise to detect low energy photons [4].

The optimal performance of such detector technologies is largely determined by the balance between the radiation absorption process and the cooling mechanism in material. In superconducting state the relaxation process, after a redistribution of the quasiparticles over energy, is determined by a recombination into Cooper pairs. The recombination time increases exponentially with decreasing temperature due to the reduced availability of quasiparticles. The scale for the recombination time is set by the electron-phonon coupling time [5]. In a normal state there are two parameters playing the main role in energy relaxation: the electron-phonon (e-ph) time and the phonon escape time. The first parameter is a property of the material, while the second is determined by the film thickness and the acoustic mismatch between the film and the substrate. Because of the crucial role of the energy relaxation processes in the devices based on nonequilibrium effects, the response of detector is strictly determined by the material dependent e-ph time.

One of the strategies for the optimization of detector performance is to select an appropriate material. The choice of the suitable material is a complex problem, and one can start with the e-ph time in the material. However, there are different requirements for different types of detectors. For SSPDs one needs materials with fast e-ph relaxation to have a fast response, materials with similar properties are required for HEB mixers to ensure a large operational bandwidth. For MKIDs and nano-HEBs, one prefers materials with long electron-phonon relaxation times to provide a high sensitivity and low noise. Another important parameter that determines the noise properties and geometry of detectors is the electron density of states, $N_{0}$. To optimize the noise properties of a detector, one chooses materials with a low $N_{0}$ [4], [6]. The final choice is a subject of the availability of a reliable technology for fabrication of thin film with desired $T_{c}$ and resistivity. For sensitive superconducting detectors, one of the important issues is to achieve controlable tuning of the critical temperature of the material to the desired value.

As a potential material for radiation detection we have studied superconducting diamond films. A significant interest to diamond for the development of electronic devices is due to the evolution of its properties with the boron dopant concentration. Boron dopants form an acceptor level that is located approximately $370 \mathrm{meV}$ above the top of the valence band. The valence band is separated from the conduction band of diamond by the wide energy gap with $E_{g} \sim 5.5 \mathrm{eV}$. For boron dopant con- 
centrations of $n_{B} \sim 10^{17} \mathrm{~cm}^{-3}$, the material is semiconducting. When the boron concentration exceeds the critical value $n_{C} \sim 10^{20} \mathrm{~cm}^{-3}$, diamond shows metallic behavior. Recently, diamond has been reported to become a superconducting at a high boron doping concentration $n_{B} \sim 5 \times 10^{20} \mathrm{~cm}^{-3}$ [7], [8]. The temperature of the superconducting transition increases with the carrier concentration, and can be varied between $1 \mathrm{~K}$ and $10 \mathrm{~K}$ by controlling the dopant level [9], [10]. The discovery of superconductivity in boron-doped diamond has opened the possibility to combine its outstanding mechanical and optical properties with superconductivity, which makes it a promising material for fully integrated hybrid systems [11], [12]. Our previous study of energy relaxation in single-crystalline borondoped diamond films epitaxially grown on a diamond shows a remarkably slow energy-relaxation at low temperatures [13]. The electron-phonon cooling time varies from $400 \mathrm{~ns}$ to $700 \mathrm{~ns}$ over the temperature range $2.2 \mathrm{~K}$ to $1.7 \mathrm{~K}$. However, the price and the small substrate growth area limit practical applications of homoepitaxial diamond films. As an alternative way with more convenient technology, one can employ heteroepitaxial diamond films grown on large-size Si substrates [14]. In this paper we report about the measurements of electron-phonon cooling times in superconducting boron-doped diamond films grown on silicon substrates and discuss our expectations about the applicability of boron-doped diamond films to superconducting detectors.

\section{DETERMINATION OF ENERGY RELAXATION TIME IN SC FILMS}

To determine the energy-relaxation time we have used the method based on amplitude-modulated absorption of sub- $\mathrm{THz}$ radiation (AMAR-method) [15]. The details of this method and conditions of its applicability are discussed in Ref [13]. The superconducting film is kept at the superconducting transition temperature, biased with a small DC current, and exposed to amplitude-modulated radiation of sub- $\mathrm{THz}$ range. The absorption of incident radiation power causes an increase of the electron temperature and consequently results in an increase of the sample resistance producing the change of the voltage signal. The amplitude of the voltage signal $\delta \mathrm{U}(\mathrm{f})$ exhibits a frequency-dependent rolloff (inset on Fig. (1)), which is used as an indicator of the response time, which is related to the intrinsic energy relaxation time. Description of the AMAR-method based on the two-temperature model, in which the electron and phonon subsystem are described in terms of two different temperatures, $T_{e}$ and $T_{\mathrm{ph}}$, respectively, whose changes are ruled by both the electronic and lattice specific heats $c_{e}$ and $c_{\mathrm{ph}}$. Electrons and phonons in the metal film are coupled via electron-phonon interaction. Assuming that the escape time of nonequilibrium phonons into the substrate could be very short, considerably less than the experimentally observed relaxation times, the phonons can be treated as a heat bath in equilibrium with the cryogenic environment. Then, in the quasiequilibrium regime, the thermal transport in the film can be described by a single heat-balance equation:

$$
c_{e} \frac{d T_{e}}{d t}=-G\left(T_{e}-T_{b}\right)+P_{\mathrm{DC}}+P_{\mathrm{RF}},
$$

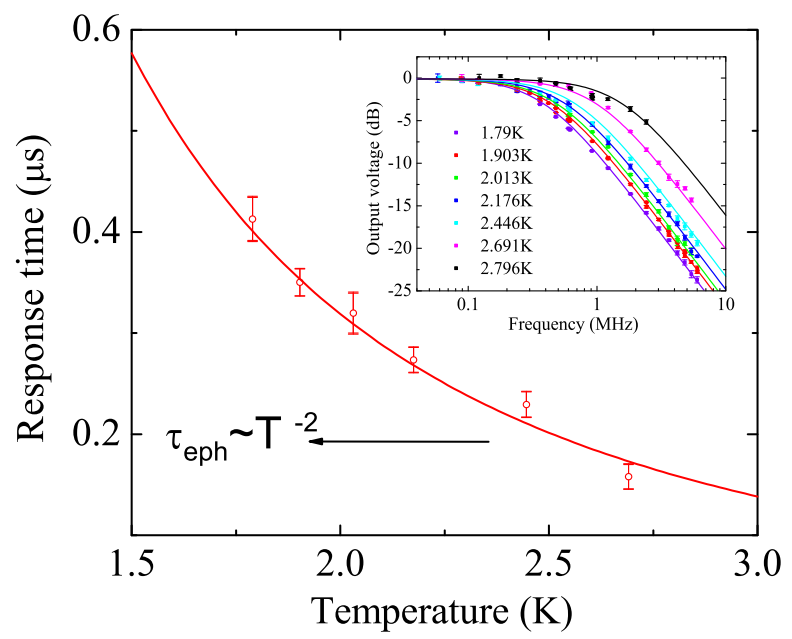

Fig. 1 The energy relaxation time for studied sample at different temperatures. The fitting line corresponds to the power law dependence for $\tau_{\text {eph }}(T)$ with exponent $p=2$. The insert shows the frequency dependencies of the response voltage at different temperatures for Sample. The data are normalized to the $0 \mathrm{~dB}$-level for convenience. The solid lines correspond to the least-square fit with Eq. (2).

where $G$ is the heat conductance from the electrons to the phonon-bath, $P_{\mathrm{DC}}=I^{2} R$ is the Joule power dissipated in the film, and $P_{\mathrm{RF}}$ is the absorbed radiation power. Because of such a simplified description, the energy relaxation time $\tau$ can be determined from the 3-dB rolloff of the frequency dependence of the amplitude of the signal:

$$
\delta U\left(f_{m}\right)=\delta U(0)\left(1+\frac{f_{m}^{2}}{f_{3 \mathrm{~dB}}^{2}}\right)^{-1 / 2}
$$

with the fitting parameters $\delta U(0)$ and $f_{3 \mathrm{~dB}}=1 /(2 \pi \tau)$ corresponding to the $3-\mathrm{dB}$ rolloff frequency of the response. The response voltage $\delta U\left(f_{m}\right)$ and the frequency $f_{m}$ are measured with a spectrum analyzer. As a radiation source, we use a backward-wave oscillator (BWO) with a carrier frequency of $350 \mathrm{GHz}$. The BWO power is amplitude-modulated at a frequency swept from 10 to $6000 \mathrm{kHz}$. To determine the temperature dependence of the relaxation time we vary the bath temperature and apply a perpendicular magnetic field to get into a usable superconducting transition at temperatures lower $T_{c}$.

\section{ELECTRON-PHONON COOLING TIME IN NANOCRYSTALLINE BORON-DOPED DIAMOND}

Nanocrystalline superconducting boron-doped diamond films are grown on non-diamond substrates by microwave plasmaenhanced chemical vapor deposition (MPECVD) [14]. Prior to the growth, a nanodiamond powder is added onto the sample substrates to provide nucleation centers for the formation of grains. In the growth chamber, microwave plasma is supplied to $\mathrm{CH}_{4} / \mathrm{H}_{2}$ precursor gas to separate the molecules into reactive radicals (the radical methyl, $\mathrm{CH}_{3}$ ), which diffuses and reacts onto the substrate surface. Hydrogen-rich gas phases (with 3\% $\mathrm{CH}_{4}$ diluted in $\mathrm{H}_{2}$ ) are used to obtain diamond films with a nanocrystalline structure. During the fabrication process, boron atom impurities are also incorporated into the diamond film 
by means of a trimethylboron gas. The details of the growth procedure are presented in Ref. [14]. The studied sample is a $520 \mathrm{~nm}$-thick diamond film was grown on a (100)-silicon substrate with a molar concentration $\mathrm{B} / \mathrm{C}$ of $6087 \mathrm{ppm}$ in the gas phase and the substrate temperature about $900{ }^{\circ} \mathrm{C}$. Its normal state resistivity was about is $2500 \mu \Omega \cdot \mathrm{cm}$, the superconducting transition occurs at $T_{c}=2.8 \mathrm{~K}$ (with the transition width of $\Delta T_{c}=0.34 \mathrm{~K}$ ). We define the critical temperature $T_{c}$ as the temperature of the midpoint of the resistive transition where the sample resistance is $50 \%$ of $R_{N}$. We have also determined the electron diffusion constant $D=0.6 \mathrm{~cm}^{2} / \mathrm{s}$ by using the temperature dependence of the second critical magnetic field (the description can be found in [13]). The frequency dependences of the response voltage $\delta U\left(f_{m}\right)$ at different temperatures are presented in the inset of Fig. (1). The bath temperature is varied between $1.7 \mathrm{~K}$ and $T_{c}=2.8 \mathrm{~K}$ by means of a perpendicular magnetic field in the range of $0-1.2 \mathrm{~T}$. By increasing the modulation frequency we find for each bath temperature a clear rolloff of the response voltage. We apply a least-square fit to the measured data using Eq. (2), which leads to a characteristic relaxation time, shown in Fig. (1). The values of $\tau(T)$ vary from $158 \mathrm{~ns}$ at $2.69 \mathrm{~K}$ to $413 \mathrm{~ns}$ at $1.79 \mathrm{~K}$, that corresponds to the power law $\tau(T)=\beta T^{-p}$ with the fitting parameters $p=2.06 \pm 0.16$ and $\beta=1330 \mathrm{~ns} \times \mathrm{K}^{-\mathrm{p}}$. We identify the observed response time as the electron-phonon relaxation time. The phonon escape time can be estimated by using the expression of $\tau_{\text {esc }}=4 d /\left(\alpha u_{s}\right)$. This $\tau_{\text {esc }}$ depends on the film thickness $d$, the acoustic match between the film and the substrate $\alpha$ and the sound velocity in material $u_{s}$. Using the values of sound velocities for monocrystal diamond $\left(u_{l}=16 \times 10^{5} \mathrm{~cm} / \mathrm{s}\right.$ and $u_{t}=9.7 \times 10^{5} \mathrm{~cm} / \mathrm{s}$ [16] $)$ and the coefficient $\alpha \sim 0.63$ for the thermal boundary between diamond and silicon (the value is estimated by following to argumentation in Ref. [17]), we obtained $\tau_{\text {esc }}$ of 350 ps. The estimated value of $\tau_{\text {esc }}$ is about three orders of magnitude less than experimentally observed relaxation time. Moreover, since the sound velocity in boron-doped diamond is higher than that in the substrate, this condition allows one to avoid phonon trapping in the film caused by total internal reflection (the acoustic analogue to Snell's law in optics) and prevents energy back-flow to the electron subsystem.

Another effect that should be accounted for is phonon re-absorption. This process is described by the characteristic phonon-electron time, $\tau_{\text {phe }}$. According to the energy balance equation, $\tau_{\mathrm{phe}}=\tau_{\mathrm{eph}}\left(c_{\mathrm{ph}} / c_{e}\right)$. Since we do not know the experimental values of the electron and phonon specific heats of boron-doped diamond films, we have made an estimate of $c_{\mathrm{ph}}$ using the Debye model. The electron specific heat capacity is determined as $c_{e}=\gamma T$, where $\gamma=\left(\pi^{2} / 3\right) k_{B}^{2} N_{0}$ is the Sommerfeld constant. For C:B films the value of $\gamma=1.632 \times$ $10^{5} \mathrm{~J} \times \mathrm{cm}^{-3} \times K^{-2}$. At $T=2 \mathrm{~K}$ this gives $c_{e}=3.2 \times$ $10^{-5} \mathrm{~J} \times \mathrm{cm}^{-3} K^{-1}$ and $c_{\mathrm{ph}}=\left(\left(12 \pi^{4}\right) / 5\right) n\left(k_{B}\right)^{2}\left(T / T_{D}\right)^{3}=$ $7 \times 10^{-7} \mathrm{~J} \times \mathrm{cm}^{-3} \mathrm{~K}^{-1}\left(n=1.77 \times 10^{23} \mathrm{~cm}^{-3}\right.$ is the number density of diamond atoms and $T_{D}=1860 \mathrm{~K}$ is the Debye temperature). Thus, the ratio $c_{\mathrm{ph}} / c_{e}=0.02$ at $T=2 \mathrm{~K}$, which yields $\tau_{\text {phe }} \sim 10 \mathrm{~ns}$ for $\tau_{\mathrm{eph}}=500 \mathrm{~ns}$, which is much larger than $\tau_{\text {esc }}$, so phonon-reabsorption does not take place. Therefore, we assume that the observed response time is determined by electron-phonon interaction.
The same dependence $\tau_{\text {eph }}(T)$ was also observed for singlecrystal film [13]. However, the absolute values of $\tau_{\text {eph }}$ in the polycrystalline film are in $\sim 1.4$ factor less than in the singlecrystal film. The difference correlates with a higher value of the electron density of states at the Fermi level in polycrystalline material. ${ }^{1}$

The experimental data show the slowest e-ph relaxation in superconducting boron-doped diamond films in comparison with the most frequently used superconducting thin films at their respective $T_{c}$. The value of $\tau_{\mathrm{eph}}$ in $\mathrm{NbN}$ corresponds to $\sim 12 \mathrm{ps}$ at $T_{c}=11 \mathrm{~K}$ [18], $\sim 800 \mathrm{ps}$ at $T_{c}=3.4 \mathrm{~K}$ in WSi [19], $\sim 1 \mathrm{~ns}$ at $T_{c}=8.5 \mathrm{~K}$ in $\mathrm{Nb}$ [20], $\sim 4.5 \mathrm{~ns}$ at $T_{c}=4.5 \mathrm{~K}$ in TiN [21], $\sim 10 \mathrm{~ns}$ at $T_{c}=11.5 \mathrm{~K}$ in NbC [22]. The listed values of $\tau_{\mathrm{eph}}$ were also determined by the AMAR-method.

The electron-phonon coupling in metals is determined by the electronic and lattice properties of the material. For disordered metal this property can be described by using the following coupling parameter: $\beta=\left(\frac{2 \varepsilon_{F}}{3}\right)^{2} \frac{N_{0}}{2 \rho_{m} u^{2}}$, where $\varepsilon_{F}$ is the Fermi energy, $N_{0}$ is the electron density of states at the Fermi level, $\rho_{m}$ is the mass density and $u$ is the sound velocity [23], [24]. The boron-doped diamond films are characterized by low values of carrier density $\left(\sim 10^{21} \mathrm{~cm}^{-3}\right)$ in comparison with typical metals $\left(\sim 10^{23} \mathrm{~cm}^{-3}\right)$ that corresponds to low values of $\varepsilon_{F}$ and $N_{0}$ in this material. Moreover, the diamond lattice is characterized by extremely high values of Debye temperature $\left(\mathrm{T}_{\mathrm{D}} \sim 1860 \mathrm{~K}\right)$ and sound velocity $\left(u \sim 1610^{5} \mathrm{~cm} / \mathrm{s}\right)$ compared with other metals (where typical values of $T_{D}$ are in the range of $300 \mathrm{~K}-500 \mathrm{~K}$, and the values of sound velocities are order of $5 \cdot 10^{5} \mathrm{~cm} / \mathrm{s}$ ). All these parameters contribute to the weaker electron-phonon coupling in borondoped diamond, and, correspondingly, to the slow energyrelaxation.

Encouraged by this result we have made an estimate of the noise-equivalent power (NEP) and the energy resolution of bolometer made from superconducting diamond. The fundamental noise mechanism of such detectors, the thermal energy fluctuation, is minimized with increasing of the e-ph relaxation time due to NEP $=\sqrt{4 k_{B} T_{e}^{2} c_{e} / \tau_{\mathrm{eph}}}$. The energy resolution is estimated by using $\delta E=\mathrm{NEP} \sqrt{\tau_{\mathrm{eph}}} \approx \sqrt{4 k_{B} T_{e}^{2} c_{e}}$. Given the material resistance and the thickness of the film, we have chosen the planar dimensions so as to couple the bolometer with the $75 \Omega \log$-spiral antenna. In our estimation of limit value of the NEP is of order $10^{-17} \mathrm{~W} / \mathrm{Hz}^{1 / 2}$ at $2 \mathrm{~K}$ and the energy resolution is of $0.1 \mathrm{eV}$ that corresponds to counting single-photon up to $15 \mu \mathrm{m}$. Although this value is far yet from what might like to have for certain astronomical applications, we believe that it can be improved by a suitable fabrication process. The comparison of NEP and $\delta E$ of diamond bolometer with the parameters of a detector based on other material with the similar value of $T_{c}$ (for example, a thin TiN film with $T_{c}=2 \mathrm{~K}$ ) revealed that long e-ph relaxation time provides a good advantage only for the NEP value. Meanwhile, the energy resolution is mainly determined by the electronic properties of material, in particular the electron

\footnotetext{
${ }^{1}$ The electron density of states at the Fermi level can be estimated using expression $N_{0}=1 /\left(e^{2} \rho D\right)$. This parameter $N_{0}$ is equal to $3.1 \dot{\mathrm{c}} 10^{9} \mathrm{eV}^{-1} \mu \mathrm{m}^{-3}$ for the studied single crystal film (with the resistivity of $1500 \mu \Omega \mathrm{cm}$ and electron diffusivity $1.38 \mathrm{~cm}^{2} / \mathrm{s}$ ) and $4.2 \dot{\mathrm{c}} 10^{9} \mathrm{eV}^{-1} \mu \mathrm{m}^{-3}$ for the polycrystalline film (with the resistivity of $2500 \mu \Omega \mathrm{cm}$ and electron diffusivity $0.6 \mathrm{~cm}^{2} / \mathrm{s}$ ).
} 
diffusivity. Another useful parameter of boron-doped diamond films is high resistivity in the normal state. This property corresponds to a low value of electron density state at Fermi level and a high kinetic inductance that are important parameters for superconducting resonator detectors.

\section{CONCLUSION}

We have studied nanocrystalline boron-doped diamond films on silicon substrates by using the AMAR-method. On the practical side, the slow energy relaxation at low temperatures in boron-doped diamond, in combination with a high normal-state resistivity, confirms that this material is a good candidate for superconducting electronic applications.

\section{ACKNOWLEDGMENT}

The authors would like to thank R. Ozhegov for technical support in the measurement and processing of the experimental data. This work was implemented in the framework of the Basic Research Program, National Research University Higher School of Economics in 2016.

\section{REFERENCES}

[1] G. N. Gol'tsman et al., "Picosecond superconducting single-photon optical detector," Appl. Phys. Lett., vol. 79, pp. 705-707, 2001.

[2] P. K. Day, H. G. Leduc, B. A. Mazin, A. Vayonakis, and J. Zmuidzinas, "A broadband superconducting detector suitable for use in large arrays," Nature, vol. 425, pp. 817-821, 2003.

[3] E. M. Gershenzon, G. N. Gol'tsman, I. G. Gogidze, A. I. Eliantev, B. S. Karasik, and A. D. Semenov, "Millimeter and submillimeter range mixer based on electron heating of superconducting films in the resistive state," Sov. Phys. Supercond., vol. 3, pp. 1582-1597, 1990.

[4] J. Wei, D. Olaya, B. Karasik, S. Pereverzev, A. Sergeev, and M. Gershenson, "Ultra-sensitive hot-electron nanobolometers for terahertz astrophysics," Nature Nanotech., vol. 3, pp. 496-500, 2008.

[5] S. B. Kaplan, C. C. Chi, D. N. Langenberg, J. Chang, S. Jafarey, and D. J. Scalapino, "Quasiparticle and phonon lifetimes in superconductors," Phys. Rev. B., vol. 14, pp. 4854-4873, 1976.

[6] H. G. Leduc et al., "Titanium nitride films for ultrasensitive microresonator detectors," Appl. Phys. Lett., vol. 97, 2010, Art. no. 102509.
[7] E. A. Ekimov et al., "Superconductivity in diamond," Nature, vol. 428 , pp. 542-545, 2004.

[8] E. Bustarret et al., "Dependence of the superconducting transition temperature on the doping level in single-crystalline diamond films," Phys. Rev. Lett., vol. 93, 2004, Art. no. 237005.

[9] T. Klein et al., "Metal-insulator transition and superconductivity in borondoped diamond," Phys. Rev. B, vol. 75, 2007, Art. no. 165313.

[10] K. Ishizaka et al., "Observation of a superconducting gap in boron-doped diamond by laser-excited photoemission spectroscopy," Phys. Rev. Lett., vol. 98, 2007, Art. no. 047003

[11] Y. Shim and Ch. Tahan, "Semiconductor-inspired design principles for superconducting quantum computing," Nature Commun., vol. 7, 2016 , Art. no. 11059 .

[12] P. Rath et al., "Superconducting single-photon detectors integrated with diamond nanophotonic circuits," Light: Sci. Appl., vol. 4, 2015, Art. no. e338.

[13] A. Kardakova et al., "Relaxation of the resistive superconducting state in boron-doped diamond films," Phys. Rev. B, vol. 93, 2016, Art. no. 064506.

[14] O. Williams et al., "Growth, electronic properties and applications of nanodiamond," Diamond Related Mater, vol. 17, pp. 1080-1088, 2008.

[15] E. M. Gershenzon, M. E. Gershenzon, G. N. Gol'tsman, A. M. Lyul'kin, A. D. Semenov, and A. V. Sergeev, "Electron-phonon interaction in ultrathin Nb films," Zh. Eksp. Teor. Fiz., vol. 97, pp. 505-511, 1990.

[16] F. Guistino, J. R. Yates, I. Souza, M. L. Cohen, and S. G. Louie, "Electronphonon interaction via electronic and lattice wannier functions: superconductivity in boron-doped diamond reexamined," Phys. Rev. Lett., vol. 98, 2007, Art. no. 047005

[17] S. B. Kaplan, "Acoustic matching of superconducting films to substrates," J. Low Temp. Phys., vol. 37, pp. 343-365, 1979.

[18] Yu. Gousev et al., "Broadband ultrafast superconducting NbN detector for electromagnetic radiation," J. Appl. Phys., vol. 75, pp. 3695-3697, 1994.

[19] M. Sidorova et al., "Electron-phonon relaxation time in ultrathin tungsten silicon film," arXiv:1607.07321.

[20] E. M. Gershenzon, M. E. Gershenzon, G. N. Gol'tsman, A. M. Lyul'kin, A. D. Semenov, and A. V. Sergeev, "Electron-phonon interaction in ultrathin Nb films," Zh. Eksp. Teor. Fiz., vol. 97, pp. 505-511, 1990.

[21] A. Kardakova et al., "The electron-phonon relaxation time in thin superconducting titanium nitride films," Appl. Phys. Lett, vol. 103, 2013, Art. no. 252602

[22] K. S. Il'in et al., "Interrelation of resistivity and inelastic electron-phonon scattering rate in impure NbC films," Phys. Rev. B, vol. 57, pp. 15623 $15628,1998$.

[23] A. Schmid, "Electron-phonon interaction in impure metal," Z. Physik, vol. 259, pp. 421-436, 1973.

[24] A. Sergeev and V. Mitin, "Electron-phonon interaction in disordered conductors: static and vibrating scattering potentials," Phys. Rev. B, vol. 61, pp. 6041-6047, 2000. 